\title{
PI3K-regulated Glycine N-methyltransferase is required for the development of prostate cancer
}

\author{
Amaia Zabala-Letona ${ }^{1,2}$, Amaia Arruabarrena-Aristorena (iD ${ }^{1,3,4}$, Sonia Fernandez-Ruiz ${ }^{1,2}$, Cristina Viera ${ }^{1,2}$, Onintza Carlevaris ${ }^{1}$, \\ Amaia Ercilla ${ }^{1,2}$, Isabel Mendizabal (D) ${ }^{1,3,4}$, Teresa Martin ${ }^{1}$, Alice Macchia (D) ${ }^{1,5}$, Laura Camacho ${ }^{1,5}$, Mikel Pujana-Vaquerizo ${ }^{1}$, \\ Pilar Sanchez-Mosquera ${ }^{1}$, Verónica Torrano ${ }^{1,2,5}$, Natalia Martin-Martin ${ }^{1,2,4}$, Patricia Zuniga-Garcia (D) ${ }^{1}$, Mireia Castillo-Martin ${ }^{6}$, \\ Aitziber Ugalde-Olano ${ }^{2,7}$, Ana Loizaga-Iriarte ${ }^{2,4,8}$, Miguel Unda $\mathbb{D}^{2,4,8}$, Jose M. Mato $\mathbb{D}^{1,9}$, Edurne Berra ${ }^{1,2}$, \\ Maria L. Martinez-Chantar (iD ${ }^{1,9}$ and Arkaitz Carracedo (iD ${ }^{1,2,3,4,5 凶}$
}

(c) The Author(s) 2022

Glycine N-Methyltransferase (GNMT) is a metabolic enzyme that integrates metabolism and epigenetic regulation. The product of GNMT, sarcosine, has been proposed as a prostate cancer biomarker. This enzyme is predominantly expressed in the liver, brain, pancreas, and prostate tissue, where it exhibits distinct regulation. Whereas genetic alterations in GNMT have been associated to prostate cancer risk, its causal contribution to the development of this disease is limited to cell line-based studies and correlative human analyses. Here we integrate human studies, genetic mouse modeling, and cellular systems to characterize the regulation and function of GNMT in prostate cancer. We report that this enzyme is repressed upon activation of the oncogenic Phosphoinositide-3-kinase (PI3K) pathway, which adds complexity to its reported dependency on androgen signaling. Importantly, we demonstrate that expression of GNMT is required for the onset of invasive prostate cancer in a genetic mouse model. Altogether, our results provide further support of the heavy oncogenic signal-dependent regulation of GNMT in prostate cancer.

Oncogenesis (2022)11:10; https://doi.org/10.1038/s41389-022-00382-x

\section{INTRODUCTION}

Prostate cancer (PCa) is among the most frequent cancer types in men and accounts for a significant mortality by the disease [1]. The clinical management of this tumor type has profoundly changed with the implementation of prostate specific antigen (PSA)-based screening, minimally invasive robotic surgery, and new-generation androgen synthesis and signaling inhibitors [2, 3]. However, the molecular understanding of the disease has not yet been fully exploited for cancer stratification and therapy, as compared to other tumor types.

Prostate tumor cells rely on the abnormal activation of the PI3K pathway [1]. Large-scale genome aberration studies corroborated the initial observations related to Phosphatase And Tensin Homolog (PTEN) copy number alterations in prostate tumors [4-6]. Furthermore, mouse modeling experiments demonstrated that the prostate is exquisitely sensitive to Pten levels, and perturbation of this gene alone can unleash a cancerous phenotype [7-9]. Many effector pathways have been accounted for the action of the PI3K in prostate cancer [10]. Among these, the reprogramming of cancer metabolism has gained attention in recent years. In this regard, we have reported that a Pten-deletion based PCa mouse model recapitulates metabolic features of human $\mathrm{PCa}$, with a predominant increase in the production of polyamines that are required for the proliferation of prostate tumor cells [9]. Polyamine metabolism stems from the catabolism of methionine and arginine [11]. Methionine is transformed into S-adenosylmethionine (SAMe) by MAT enzymes, and this metabolite can follow two different fates [11]. On the one hand, demethylation of SAMe by methyltransferases leads to the production of S-adenosylhomocysteine (SAH), which can enter the trans-sulfuration or remethylation pathway. The demethylation of SAMe is carried out by a variety of enzymes, including the Glycine N-methyltransferase (GNMT) [12]. On the other hand, SAMe can be decarboxylated by Adenosylmethionine Decarboxylase 1 (AMD1) to produce dcSAMe [11]. The control of AMD1 levels and activity by mTOR complex 1 (mTORC1) provides a feasible explanation for the elevated production of polyamines upon PTEN deletion [9].

GNMT is a metabolic enzyme predominantly expressed in the liver, pancreas, brain, and prostate tissue [12]. It catalyzes the transfer of a methyl group from SAMe to Glycine, resulting in the production of SAH and sarcosine. This enzyme is a well-accepted tumor suppressor in the liver [12]. Deletion of Gnmt in the mouse results in steatosis, steatohepatitis, and development of hepatocarcinoma late in life [13]. In contrast, various reports suggest that this enzyme favors various oncogenic features in PCa cells $[14,15]$. GNMT overexpression in cell lines, or the supplementation with its

\footnotetext{
${ }^{1}$ Center for Cooperative Research in Biosciences (CIC bioGUNE), Basque Research and Technology Alliance (BRTA), Bizkaia Technology Park, Building $801 \mathrm{~A}, 48160$ Derio, Spain. ${ }^{2}$ CIBERONC, Madrid, Spain. ${ }^{3}$ kerbasque, Basque Foundation for Science, Bilbao, Spain. ${ }^{4}$ Traslational prostate cancer Research lab, CIC bioGUNE-Basurto, Biocruces Bizkaia Health Research Institute, Derio, Spain. ${ }^{5}$ Biochemistry and Molecular Biology Department, University of the Basque Country (UPV/EHU), Bilbao, Spain. ${ }^{6}$ Department of Pathology, Fundação Champalimaud, Lisboa, Portugal. ${ }^{7}$ Department of Pathology, Basurto University Hospital, 48013 Bilbao, Spain. ${ }^{8}$ Department of Urology, Basurto University Hospital, 48013 Bilbao, Spain. ${ }^{9}$ Centro de Investigación Biomédica en Red de Enfermedades Hepáticas y Digestivas (CIBERehd), Madrid, Spain. ${ }^{凶}$ email: acarracedo@cicbiogune.es
}

Received: 2 March 2021 Revised: 23 December 2021 Accepted: 24 January 2022

Published online: 23 February 2022 
product sarcosine, increases several oncogenic characteristics according to various reports [14-18]. In patients, there are conflicting results, posing GNMT as an enzyme overexpressed [14] and downregulated [19] in PCa. This discrepancy could be due to the distinct nature of the patient cohorts analyzed, or to the underlying molecular regulation of this enzyme by cancerrelevant factors such as MYC, ERG, or androgen receptor [15, 20-22]. Moreover, small nucleotide polymorphisms in GNMT locus have been associated to PCa risk [19, 23], although their functional impact on the enzyme remains obscure. Importantly, there is little evidence of the contribution of GNMT to PCa pathogenesis using immunocompetent genetic mouse models that are faithful to the human disease to date.

In this study, we provide a formal demonstration of the essential nature of GNMT expression for the development of $\mathrm{PCa}$, and uncover a new regulatory mode of this enzyme downstream the oncogenic PTEN-PI3K pathway.

\section{METHODS}

\section{Patient recruitment}

Human prostate tissue specimens were obtained from the Basque Biobank for research (BIOEF, Basurto University hospital). According to the Declaration of Helsinki principles, all participants in the study provided written informed consent to clinical investigations using an approved consent form and with evaluation and approval from the corresponding ethics committee (CEIC-E 11-12, 14-14, and 19-20). All data were doublecodified to protect the confidentiality of individual participants. Tissue was accrued and processed following the protocol reported [24]. The clinicalpathological characteristics of the cases included in this study are presented in Supplementary Table 1.

\section{Animals}

All mouse experiments were carried out following the ethical guidelines established by the Biosafety and Animal Welfare Committee at CIC bioGUNE. The procedures employed were carried out following the recommendations from AAALAC. GEMM experiments were carried out in a mixed background as reported [25]. The Pten ${ }^{\text {lox }}$ conditional knockout allele has been described elsewhere [7]. The prostate epithelium-specific deletion was effected by the $\mathrm{Pb}-\mathrm{Cre} 4$ [7]. Orchiectomy was performed in 6 months $\mathrm{Pten}^{p c-/-}$ mice, and 6 days later prostate lobules were collected. Mice were fasted for $6 \mathrm{~h}$ prior to tissue harvest in order to prevent metabolic alterations due to immediate food intake. The Gnmt knockout mouse model was previously generated and characterized [13]. These mice are whole body mutants and reportedly develop steatosis and hepatocarcinoma, in line with the predominant expression of Gnmt in this organ. No randomization was used when comparing genotypes. Castration experiments were performed as reported [26].

\section{Reagents}

Cell lines were purchased from Leibniz-Institut DSMZ-Deutsche Sammlung von Mikroorganismen und Zellkulturen GmbH (DSMZ) and tested negative for mycoplasma. Low passage stock cell lines were tested for cell identity validation. Rapamycin (prepared in DMSO, final concentration $20 \mathrm{nM}$ ), MK-2206 (prepared in DMSO, final concentration $500 \mathrm{nM}$ ), BKM120 (prepared in DMSO, final concentration $5 \mu \mathrm{M}$ ), MDV-3100 (prepared in DMSO, final concentration $10 \mu \mathrm{M}$ ), I-BET762 (prepared in DMSO, final concentration $1 \mathrm{uM})$, and $\mathrm{DHT}$ (4,5a-Dihydrotestosterone, dissolved in ethanol, final concentration $10 \mathrm{nM}$ ) were purchased from LC laboratories (Rapamycin), ShelleckChem (BKM120, MK-2206), SCBT (MDV-3100), CAYMAN (I-BET762), and Sigma-Aldrich (DHT). Doxycycline was purchased from Sigma and used at $500 \mathrm{ng} / \mathrm{mL}$ for over-expression of YFP-PTEN.

shRNAs against GNMT (TRCN0000000326: sh1; TRCN0000000329: sh4) and FOXO1 (TRCN0000039582) were purchased from Sigma and control shRNA sequence is included (CCGGCAACAAGATGAAGAGCACCAACTCG AGTTGGTGCTCTTCATCTTGTTG). YFP-PTEN lentiviral constructs were described in [27].

\section{Cellular and molecular assays}

Cell number quantification was done with crystal violet [28]. Foci assays were performed seeding 500 cells per well (6-well plate) and staining and counting them by crystal violet. Lentiviral and retroviral transductions were performed as previously described [25]. Western blot was performed as previously described [29]. The following antibodies were used for Western blotting: rabbit pAnti-RpS6 5240/244, anti-RpS6, anti-HSP90, anti-PTEN (138G6), anti-AKT ${ }^{\text {S473, }}$, anti-AKT (all dilution 1:1000) and anti-cleaved PARP (D64E10) (dilution 1:1000) were all from Cell Signalling. RNA was extracted using NucleoSpin ${ }^{\circledast}$ RNA isolation kit from Macherey-Nagel (ref: 740955.240 C). 1 ug of total RNA was used for CDNA synthesis using qScript cDNA Supermix from Quanta (ref. 95048). Quantitative Real Time PCR (q-RTPCR) was performed as previously described [29]. Applied biosystems TaqMan probes: Gnmt/GNMT (Mm00494688_m1, Hs00219089_m1), FOXO1 (Hs00231106_m1) GAPDH/ Gapdh (Hs02758991_g1/Mm99999915_g1), and B-ACTIN (Hs 99999903 m1).

\section{Immunohistochemical analysis}

After euthanasia, histochemical analysis of a hematoxylin and eosin (H\&E)-stained section from formalin-fixed paraffin-embedded prostate tissues was performed as previously described [25]. Scoring system performed by pathologist was based on: Normal (0); Increased cellularity (+1); Low-grade prostate intraepithelial neoplasia (LGPIN) (+2); High-grade PIN (HGPIN) (+3); Cancer $(+4)$.

\section{Metabolomic analysis}

For in vitro metabolomic analysis, growing cells were washed with ammonium carbonate $\mathrm{pH} 7.4$ and snap-frozen in liquid nitrogen. Metabolites were extracted from cells or tissues with cold 80/20 (v/v) methanol/water. Samples were then dried and stored at $-80^{\circ} \mathrm{C}$ until MS analysis. Quantitative LC/MS was conducted as previously described [30] at AGIOS Pharmaceuticals. A Thermo Accela 1250 pump delivered a gradient of $0.025 \%$ heptafluorobutyric acid, $0.1 \%$ formic acid in the water, and acetonitrile at $400 \mu \mathrm{L} / \mathrm{min}$. The stationary phase was an Atlantis T3, $3 \mu \mathrm{m}$, $2.1 \times 150 \mathrm{~mm}$ column. A QExactive Mass Spectrometer was used at 70.000 resolving power to acquire data in full-scan mode. Data analysis was conducted in MAVEN [31] and Spotfire. Peak areas derived from stable isotope labelling experiments have been corrected for naturally occurring isotope abundance.

\section{ENCODE visualization}

FOXO1 ChIP-Seq data of two replicates ("bed narrowPeak conservative IDR thresholded peaks" files from experiment ENCSR3210AA) were downloaded from https://www.encodeproject.org/experiments/ENCSR321OAA/. ENCODE Candidate Cis-Regulatory Elements (cCREs) EH38E2468125, EH38E2468126 and EH38E2468127 were represented in the Figure. Gviz $R$ package [32] was used for visualization.

\section{Gene expression analyses from patient datasets}

Data were obtained from the public repository GEO [33], and CBioPortal $[34,35]$. All the datasets used, except Varambally [36], were downloaded as normalized data, which was corroborated by performing quality controls by means of boxplots and principal component analysis (PCA). For Varambally, a normalization by quartiles was applied [37] and a log2 transformation was performed. In addition, probes from this dataset that could lead to error due to cross-hybridizations were removed.

For gene expression comparisons among groups Student $T$ test or ANOVA was performed as indicated. For pairwise correlations between the expression of selected genes, primary tumor samples were selected, and the Pearson correlation coefficient and $p$ value was calculated as indicated in the graphs.

\section{Statistical analysis}

No statistics were applied to determine the sample size. Mice for in vivo analyses were selected based on genotype, without randomization required. For castration experiments, mice of the selected genotype were randomly assigned to the experimental groups. The investigators were not blinded to allocation during experiments and outcome assessment. Data analysed by parametric tests are represented by the mean \pm s.e.m. of pooled experiments and for non-parametric tests median with interquartile range is depicted, unless otherwise stated. $\mathrm{n}$ values represent the number of independent experiments performed or the number of individual mice or patient specimens. For each independent in vitro experiment, at least two technical replicates were used and a minimum number of three independent experiments were performed to ensure adequate statistical power. Student's $t$-test was used for two-component comparisons. In the 
in vitro experiments, normal distribution was confirmed or assumed (for $n<5$ ). Variance differences were analysed in comparisons between groups. For two-group analyses, Welch correction was applied for heteroscedastic models, and one-sample $t$-test analysis when one of the groups lacked variance (reference values). Two-tailed statistical analysis was applied for experimental design without predicted result, and one-tail for validation or hypothesis-driven experiments. The confidence level used for all the statistical analyses was of 0.95 (alpha value $=0.05$ ).

\section{RESULTS}

To study the role of GNMT in PCa, we took advantage of a mouse model faithful to the human disease, the Pten conditional knockout mice, that we have previously characterized [7-9]. We analyzed prostate tissue from Pten prostate conditional knockout mice at the onset of invasive prostate carcinoma $(\mathrm{PCa}, 6$ months old) [7]. To our surprise, cancerous lesions were accompanied by a profound reduction in Gnmt expression (Fig. 1A). We reasoned that this unexpected result could derive from the strong Pten lossdependent PI3K stimulation, since this model has demonstrated high potential to uncover PI3K signaling-dependent molecular regulations [9]. PTEN is a negative regulator of PI3K signaling, which controls cancer cell biology at multiple levels [10]. To address this notion, we studied GNMT expression levels in human PCa specimens in which PTEN status was annotated [6, 38]. Importantly, GNMT mRNA levels were significantly lower in prostate tumors with PTEN deletion in two independent prostate cancer datasets $[6,38]$, thus supporting the results of our mouse model (Fig. 1B). To understand the signaling interplay and the mechanistic basis of PTEN-regulated GNMT expression, we employed genetic and pharmacological modulation in PC3 and LNCaP prostate cancer cell lines that present a complete loss of PTEN. In order to ascertain which components of this pathway are needed for the regulation of GNMT, we inhibited various proximal and distal effectors of PI3K, using BKM120 (BKM, Pan-PI3K inhibitor), MK2206 (MK, AKT inhibitor), and Rapamycin (R, mTOR complex 1 -mTORC1- inhibitor) (Supplementary Fig. S1A, B) [9]. Interestingly, drug treatment in two PTEN-deficient PCa cell lines revealed that GNMT expression was responsive to PI3K and AKT inhibition, but not to the blockade of mTORC1 (Fig. 1C, Supplementary Fig. S1C). Despite our efforts to detect GNMT protein levels, the antibodies tested exhibited limited sensitivity for endogenous GNMT in human prostate specimens, compared to ectopically-expressed protein (Data not shown).

AKT regulates the nuclear localization of the FOXO family of transcription factors [39]. Interestingly, the regulation of GNMT by FOXO has been reported in cells from different organisms,
A

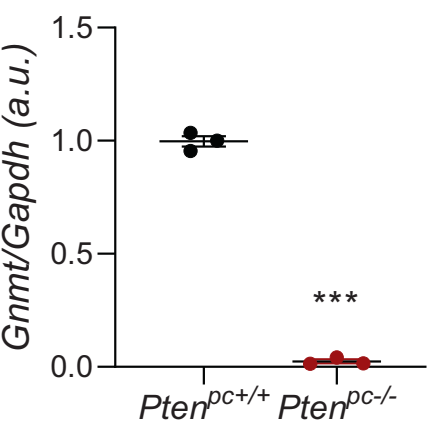

C

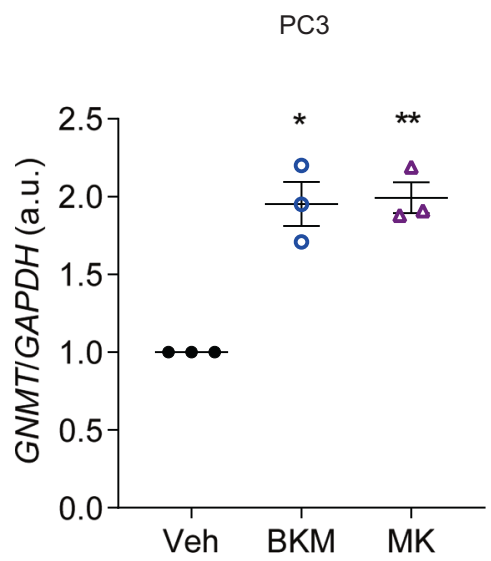

TCGA

p-value $<0.0001$

Mann-Whitney
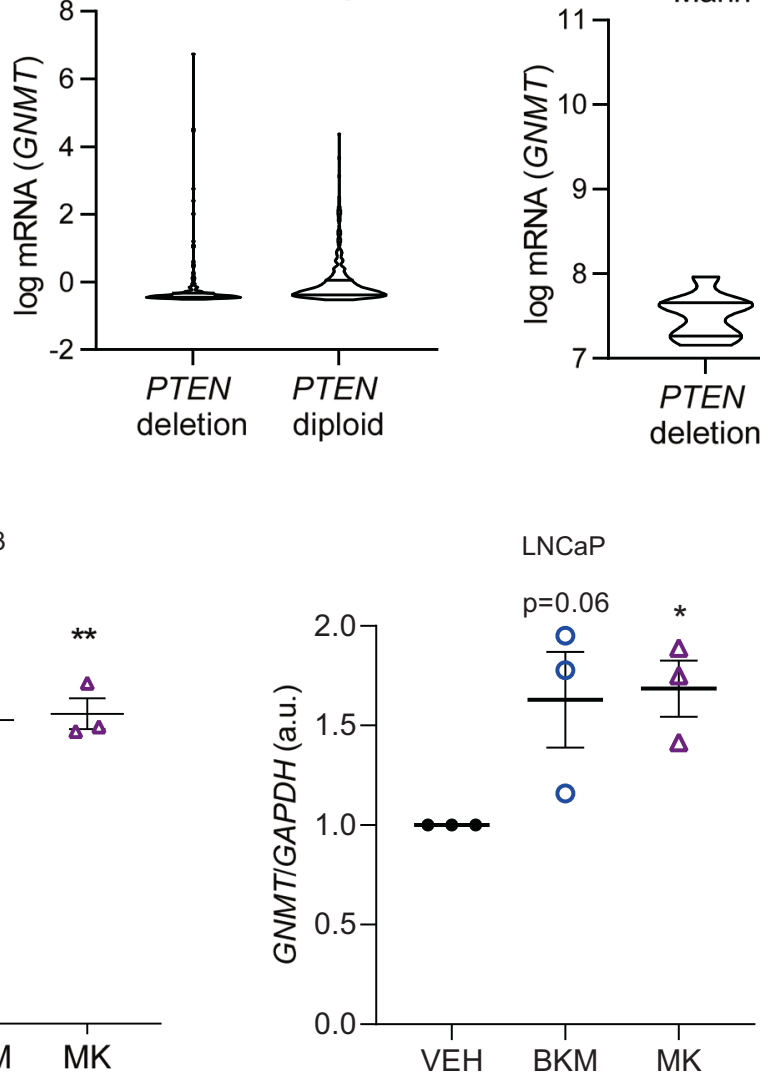

Fig. 1 PTEN regulates GNMT expression. A Gnmt mRNA expression in $P$ ten $^{p c-/-}$ and $P$ ten ${ }^{p c+/+}$ prostate tissue from 6-month-old mice $(n=3)$. B GNMT expression in PCa patient specimens with diploid or homozygous deletion of PTEN in two different datasets. Data was retrieved from www.cbioportal.org. C GNMT mRNA expression upon treatment (24h) with Vehicle (Veh, DMSO), BKM120 (BKM, 5 HM) and MK2206 (MK, $500 \mathrm{nM})$ in PC3 and LnCaP cells $(n=3)$. Data are represented as mean with SEM $(\mathbf{A}, \mathbf{C})$ and violin plot (B). Statistic test: Student $T$-test $(\mathbf{A})$; Mann-Whitney $U$-test (B) and One sample $T$-test (C). $p, p$ value; ${ }^{*} p<0.05 ;{ }^{* *} p<0.01 ;{ }^{* * *} p<0.001$. 
including the fruit fly and mouse models $[40,41]$. We interrogated available chromatin immunoprecipitation (ChIP) data to further assess the binding of FOXO family factors to GNMT regulatory regions. The ENCODE consortium has generated a variety of resources using CRISPR-Cas9 technology to introduce ChIPcompatible tags prior to exon 1 of transcription factors and regulators [42]. Interestingly, ChIP-Seq data from tagged FOXO1 was available in ENCODE for the human HepG2 cell line. The results confirmed the presence of two putative binding sequences of FOXO1 in the GNMT promoter region (Supplementary Fig. 1D). To extend the implication of the chromatin-binding capacity of FOXO1 to GNMT promoter, we performed silencing experiments using FOXO1-targeting short hairpin RNA. As predicted, FOXO1 silencing significantly reduced the mRNA abundance of GNMT in human PC3 cells (Supplementary Fig. S1E). These results corroborate that AKT-FOXO1 axis controls, at least in part, the expression of GNMT in prostate cancer cells.

We next evaluated whether the regulation of GNMT by PI3K could be translated to non-stratified prostate cancer specimens. We interrogated a local cohort of benign prostate hyperplasia (BPH) and PCa human specimens [9] (clinical-pathological information available in Supplementary Table 1) and publicly available PCa datasets using Cancertool [43]. To our surprise, independent analysis of various cohorts revealed a profound lack of consistency, yielding no significant trend in GNMT expression in prostate tumors vs. noncancerous tissue (Supplementary Fig. S2A-E), neither when comparing with normal tissue, when discriminating epithelial and stromal compartments [44] nor when accounting for tumor aggressiveness.

The lack of consistency between our mechanistic findings and the clinical evidence pointed at the existence of other regulators of GNMT expression at play in PCa. We interrogated two main potential GNMT regulators, MYC and AR. On the one hand, we found that in vitro MYC inhibition using iBET [45] led to GNMT upregulation in two different cell lines (Supplementary Fig. S3A, B). These results are in agreement with studies reporting that this oncogene inhibits the expression of GNMT [20]. However, this level of regulation was not translated to an inverse correlation between MYC and GNMT in patient specimens (Supplementary Fig. S3C) [43]. On the other hand, we ascertained the influence of AR activation status on the expression of GNMT. We subjected ARexpressing (LNCaP) and non-expressing (PC3) cells to AR agonist and antagonist treatment. The evaluation of GNMT mRNA levels upon these perturbations confirmed that the expression of this enzyme is robustly activated by the nuclear receptor. LNCaP cells exhibited GNMT upregulation and repression upon treatment with Dihydrotestosterone (DHT) and MDV3100, respectively (Fig. 2A, B). In contrast, GNMT expression in PC3 cells remained unaffected upon exposure to these agents (Supplementary Fig. S4A). Furthermore, surgical castration in prostate-specific Pten-deficient mice with prostate cancer led to a profound decrease of Gnmt mRNA levels, consistent with the repression of a bona fide AR target, $N k x 3.1$ (Fig. 2C, D). AR activity (by means of the expression of its target $K L K 3$ ) was consistently correlated with the expression of GNMT in the majority of prostate cancer datasets analyzed (Supplementary Fig. S4B, C). However, we did not find a significant correlation between tumoral GNMT expression and circulating PSA levels, in line with the notion that blood PSA is associated to prostate mass rather than AR activity (Supplementary Fig. S4C).

The abundance of GNMT and KLK3 mRNA was significantly higher in AR-expressing cell lines (22RV1, LnCaP, and C4-2), and both genes exhibited significant correlation (Fig. 2E, Supplementary Fig. S4D, E). We exploited these data further to confirm the results obtained through pharmacological and genetic means. We monitored the impact of PTEN status on GNMT expression in cell lines, to which end we focused on AR-negative cells to eliminate the dominant influence of androgen signaling. As predicted, PTENdeficient PC3 cells exhibited lower GNMT mRNA abundance than PTEN-competent DU145 (GNMT expression in arbitrary units of
$1.06 \pm 0.27$ in PC3 compared to $1.82 \pm 0.49$ in DU145; one-tailed Student $T$-test $p=0.039$ ). In line with this notion, we observed that the re-expression of PTEN in LNCaP cells resulted in elevated GNMT expression, but this effect was abolished when AR was inhibited (Fig. 2F, Supplementary Fig. S5). Our results add complexity to the regulation of GNMT in PCa.

Independent studies suggest that GNMT either promotes or suppresses PCa aggressiveness [14, 15, 17, 19]. The opposing regulation of this metabolic enzyme by androgens, MYC, and PI3K made difficult to anticipate its role in PCa. The activation of GNMT expression by androgens would be consistent with a tumorpromoting role, whereas the inhibition by the MYC and PI3K pathways would argue in the opposite direction. We chose to experimentally discern its contribution to various aspects of $\mathrm{PCa}$ biology. To this end, we took advantage of a germline Gnmt-deficient mouse model [13] and evaluated the impact of deleting this enzyme on prostate histology. The evaluation of Gnmt knockout mice for up to 1-year did not reveal neither changes in prostate mass nor pathological alterations compatible with cancer (Supplementary Fig. $\mathrm{S} 6 \mathrm{~A}-\mathrm{C})$. In line with this notion, we did not detect any significant metabolic alterations in the prostate tissue of these mice, neither at 8 months of age (Supplementary Fig. S6D and Supplementary Table 2) nor at the experimental end point (12 months of age, Supplementary Table 3). Similarly, Gnmt deletion did not impact on the prostate mass or pathology of mice in a prostate conditional Pten heterozygous background. Pten heterozygous mice exhibited PIN lesions at low incidence, as reported (Fig. 3A, B, Supplementary Fig. S7A) [25], and this phenotype remained unmodified when Gnmt was deleted. Interestingly, we found a reduction in sarcosine abundance in Gnmt-deleted tissues, and a significant increase in betaine (Fig. 3C; Supplementary Fig. S7B; Supplementary Table 4 for additional metabolites measured). In line with our previous work and other studies, conditional homozygous Pten deletion in the prostate epithelium resulted in the development of PCa [25]. This phenotype was fully penetrant in this mixed genetic background and at 12 months of age. Gnmt deletion in this model resulted in a distinct histological phenotype, with a remarkable decrease in the incidence of adenocarcinoma (Fig. 3D-F; Supplementary Fig. S8A). Pten-Gnmt double knockout mice retained PIN lesions that did not progress to cancer, whereas the overall tissue weight remained unaffected. At the metabolic level, Gnmt deletion resulted in a modest reduction in prostatic sarcosine levels (Fig. 3G) together with a significant perturbation of putrescine and methionine (Supplementary Fig. S8B; Supplementary Table 5 for additional metabolites measured).

The results in vivo are derived from a mouse model in which Gnmt was deleted systemically. In order to address whether the effect of Gnmt deletion was based on prostate cancer cellautonomous effects, we silenced GNMT in three independent PCa cell lines with differing AR status and GNMT levels (PC3, DU145, and $\mathrm{LNCaP}$ ) using two different short hairpin RNAs (Fig. $4 \mathrm{~A}-\mathrm{H}$; Supplementary Fig. S9A-D). GNMT silencing had a significant impact on cell number, with a reduction in two-dimensional growth and foci formation (Fig. 4B, C, F, G; Supplementary Fig. $\mathrm{S} 9 \mathrm{~B})$. In addition, we could confirm that the reduction in GNMT levels resulted in an overall decrease in its metabolic product, sarcosine, in PC3 and DU145 cells (Fig. 4D, H), in the absence of consistent alterations in a large panel of metabolites (Supplementary Fig. S9C). Of note, GNMT silencing elicited an increase in the apoptotic marker cleaved PARP in LnCaP and PC3 cells, in line with previous reports [46] (Supplementary Fig S9D). Altogether, the results in cell lines corroborate the in vivo results and demonstrate the requirement of GNMT for the proper maintenance of tumorigenic features.

\section{DISCUSSION}

Experimental systems are instrumental to provide a good understanding of biological processes relevant to human health. 
A

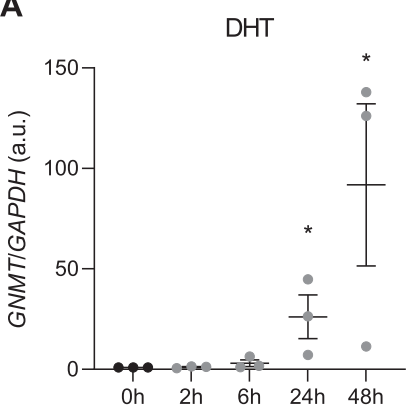

C

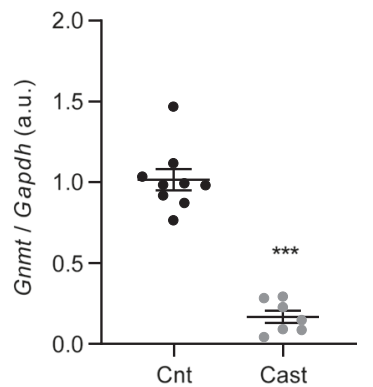

E

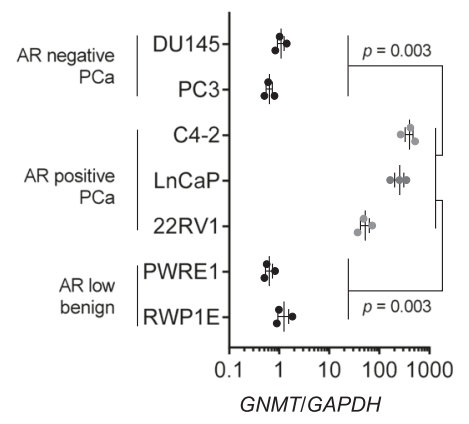

B

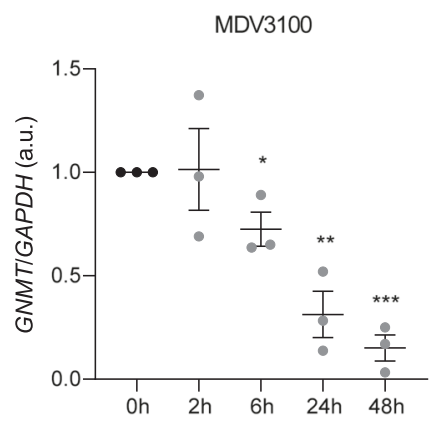

D

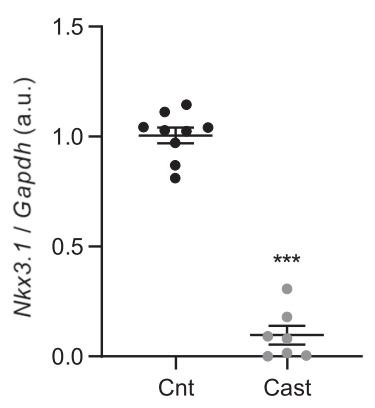

$\mathbf{F}$

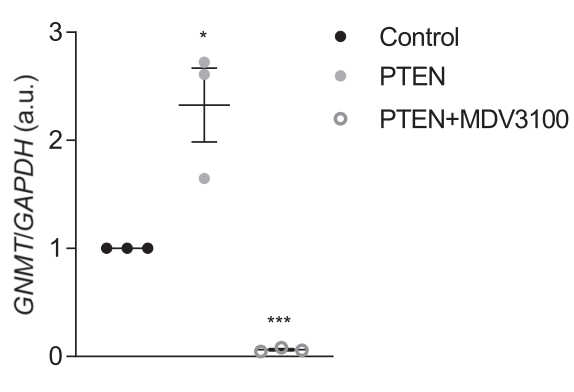

Fig. 2 GNMT regulation by AR. A-B Effect of AR activation with $5 \alpha$-dihydrotestosterone (DHT) (A) and AR inhibition by MDV3100 (B) on GNMT mRNA levels in a time course in LNCaP cells. C-D Effect of surgical castration of 6-month old Pten ${ }^{\text {pc-l- }}$ mice on Gnmt (C) and Nkx3.1 (D) mRNA expression monitored by qPCR $(n=8-10)$ (Cnt Control non-castrated, Cast Castrated). E GNMT mRNA levels in prostate cancer cell lines with AR activity (represented with grey dots) and with low or no activity (black dots) $(n=3)$. F GNMT mRNA expression upon doxycycline-inducible expression $(24 \mathrm{~h})$ of YFP-PTEN in PTEN-deficient LNCaP prostate cancer cells in the presence or absence of the AR antagonist MDV3100 $(n=3)$. Values are represented as mean with SEM. Statistics: One sample T-test (A, B, F); Mann-Whitney $U$-test $(\mathbf{C}, \mathbf{D})$ and Student $T$-test with Welch correction (E). $p, p$ value; ${ }^{*} p<0.05 ;{ }^{* *} p<0.01 ;{ }^{* * *} p<0.001$.

However, different approaches can yield apparently conflicting results. In the context of GNMT, there is sufficient evidence to support its regulation by androgen signaling [21, 22]. In addition, in vitro and xenograft approaches provided support for a positive role of GNMT in the regulation of specific PCa features [14, 15], despite the fact that some studies raised controversy around its potential tumor-suppressive nature [19]. Similarly, the analysis of human specimens has resulted in discordant observations, from upregulation in PCa [14], to downregulation in cancerous specimens [19]. Another line of evidence suggests that polymorphisms in GNMT gene are associated to higher risk of suffering $\mathrm{PCa}$ $[19,23]$, although the functional consequences of those genetic alterations remain obscure. Our work provides a comprehensive analysis of the role and regulation of GNMT in PCa. We integrate and rationalize the mechanism behind the discordant regulation of this enzyme in human and murine specimens of PCa. In addition, we provide solid evidence of the requirement of GNMT for the development of PCa using genetic mouse models complemented with genetic manipulation in cellular systems.

The regulation of GNMT in PCa is dominated by the action of AR. This gene contains androgen response elements (AREs) that makes it responsive to the activity of the nuclear receptor $[15,21,22]$. Yet, this enzyme is not consistently upregulated in PCa according to our comprehensive analysis, which adds complexity to the already contradictory published reports $[14,19]$. Our results depicting the strong correlation between GNMT and KLK3, together with the characteristic pattern of expression of this enzyme in prostate cell lines supports the notion that androgens are the main signal controlling GNMT expression, but this level of regulation co-exists with the action of other oncogenic signaling pathways (Fig. 5).

Our study demonstrates that other PCa-relevant signals influence GNMT expression. On the one hand, we observed an upregulation of this enzyme upon pharmacological blockade of MYC signaling with 
A

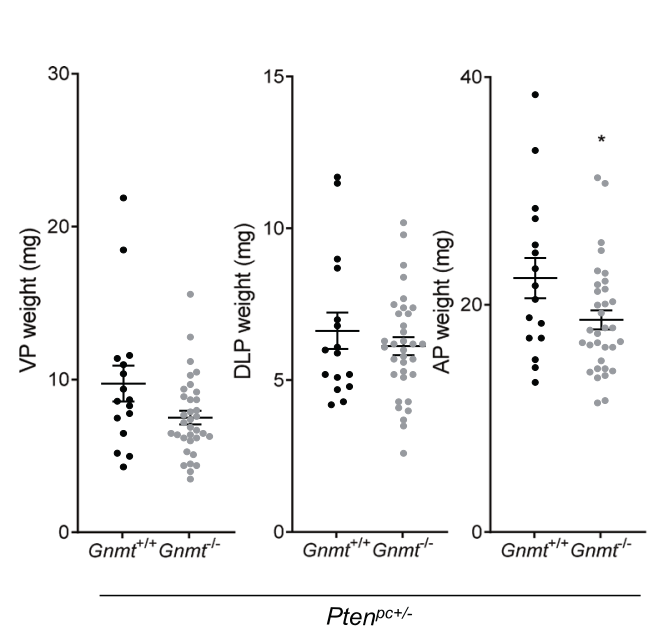

D

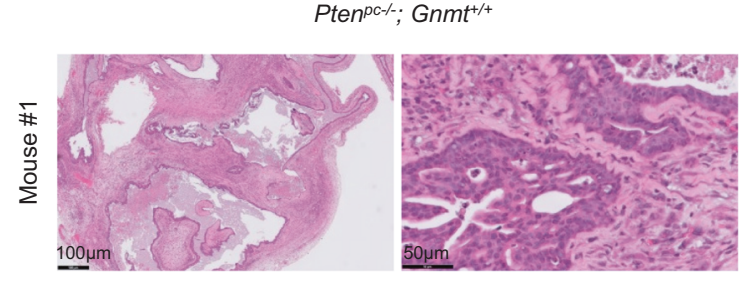

B

C
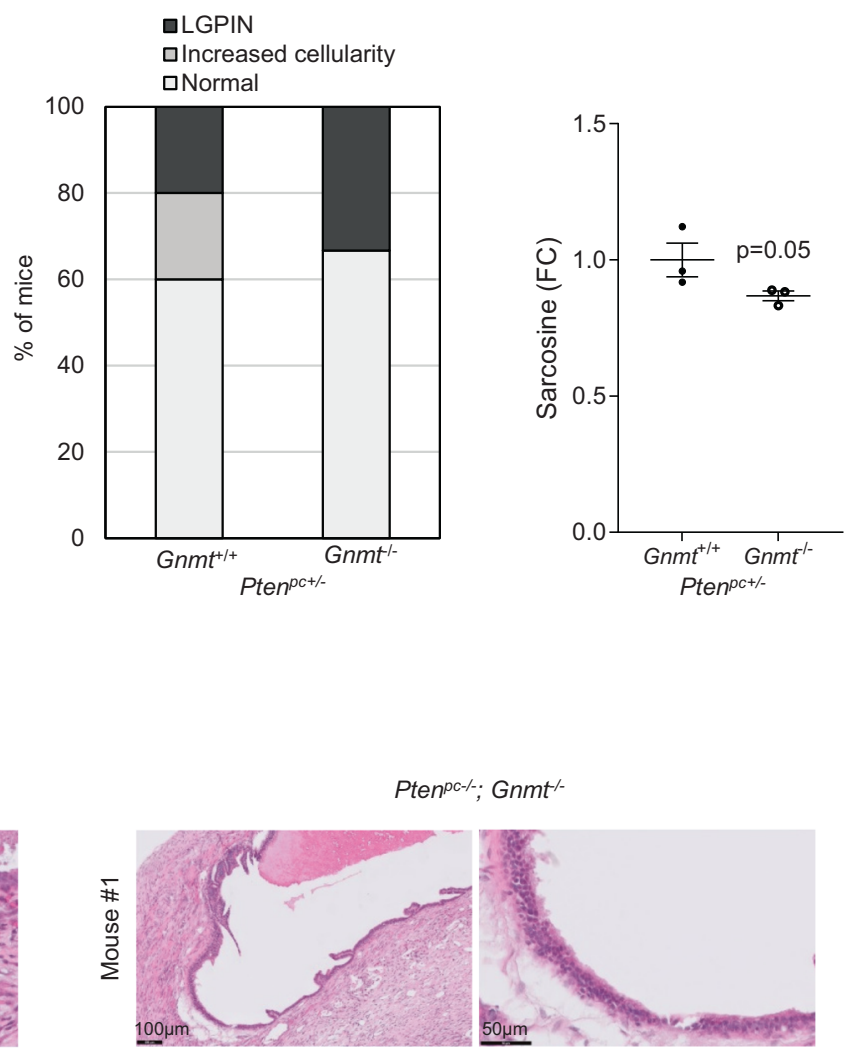

E

$\mathbf{F}$

G
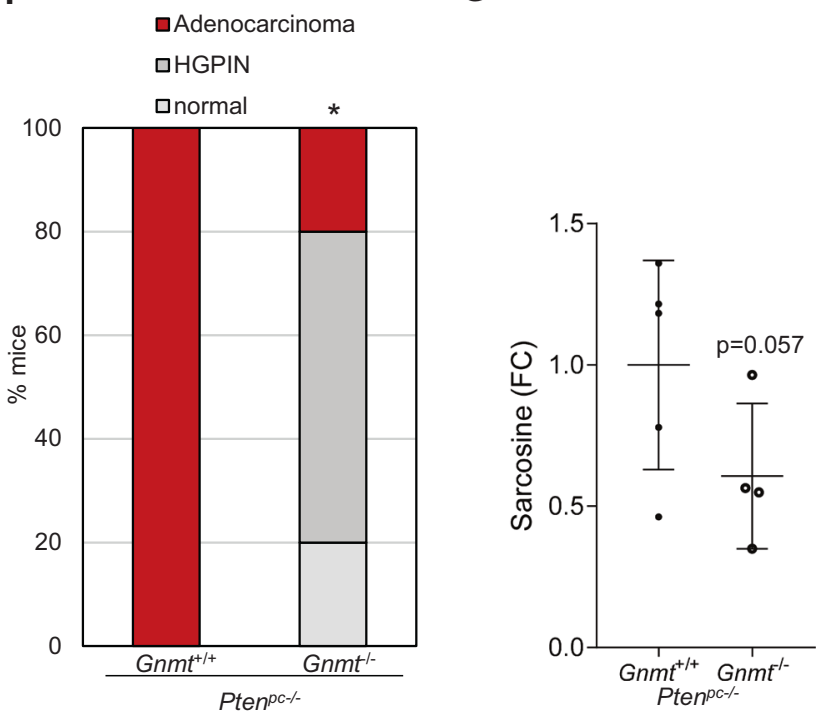

Fig. 3 Gnmt is required for prostate cancer development. A-C Prostate lobe weights (VP, DLP, and AP) in Pten ${ }^{p c+/-} ; G^{n} t^{+/+}$and Pten ${ }^{p c+/-}$; $\mathrm{Gnmt}^{-1-}$ mice (2 individual lobes are presented per mouse, $\left.n=8-16\right)(\mathbf{A})$; Histopathological characterization of the prostate tissue in Pten ${ }^{p c+1-}$; $\mathrm{Gnmt}^{+/+}$and Pten ${ }^{p+1-} ; \mathrm{Gnmt}^{-1-}$ mice $(n=8-16)(\mathbf{B}) ;$ Sarcosine abundance in anterior prostate (AP) lobes in the indicated genotypes measured by LC/MS $(n=3)(\mathbf{C})$. D-G Representative histological images of Hematoxylin Eosin (H\&E) staining (100X and 400X) in Pten ${ }^{p c-l-} ;$ Gnmt $^{+/+}$and Pten ${ }^{p c-l-}$; $\mathrm{Gnmt}^{-1}$ mouse prostates (D); Comparison of different prostate lobe weights (VP, DLP, and AP) (represented in the graph with 2 different individual lobes) $(n=4-5)$ Pten $^{p c-l-} ; G_{n m t}{ }^{+/+}$and Pten ${ }^{p c-l-} ; G_{n m t}{ }^{-1-}$ mice (E); Histopathological characterization of the prostate tissue $(n=5)(\mathbf{F})$ and Sarcosine amount in anterior prostate (AP) lobes measured by LC/MS $(n=4-5)(\mathbf{G})$ in Pten ${ }^{p c-l-} ; G_{n m t}^{+/+}$and Pten ${ }^{p c-1-} ; G n m t^{-l-}$ mice $(n=5)$. VP Ventral prostate, DLP Dorsolateral prostate, AP Anterior prostate, LGPIN Low-grade Prostatic Intraepithelial Neoplasia, HGPIN High grade Prostatic Intraepithelial Neoplasia. Statistics: One tail Mann-Whitney $U$ test (A left and middle panels, C, E, G), Two tailed Student $T$ test (right panel), and Chi square (F) was used for data analysis. $p, p$ value; ${ }^{*} p<0.05$. 
A

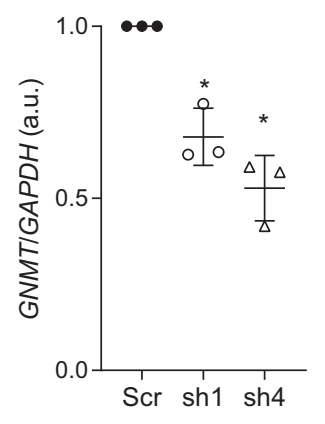

B

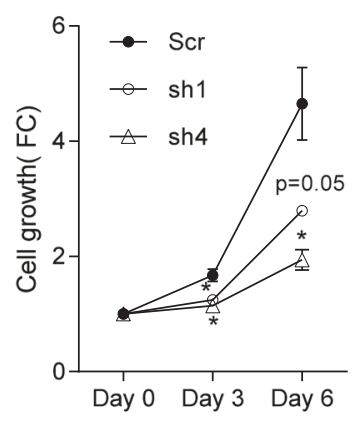

C

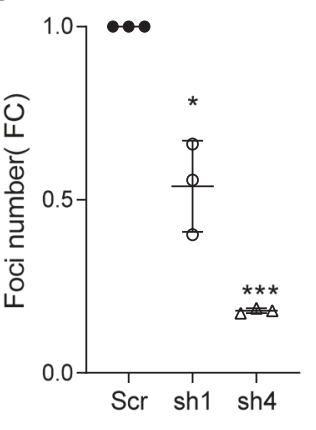

D

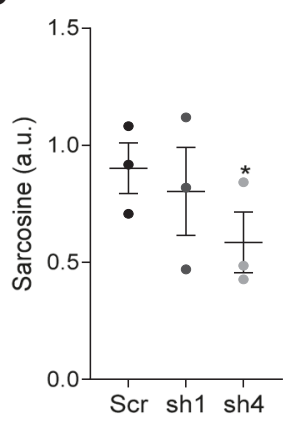

PC3 shGNMT

E

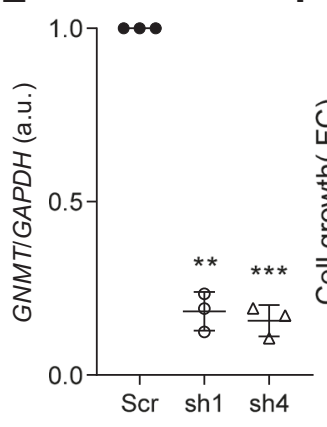

F

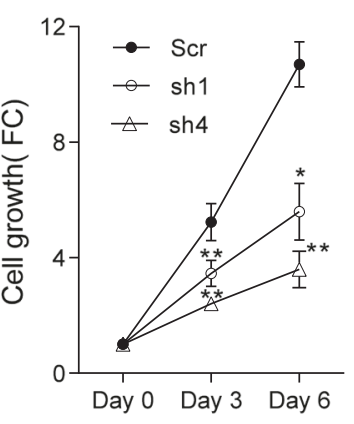

G

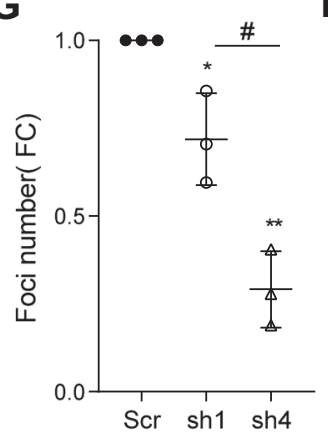

H

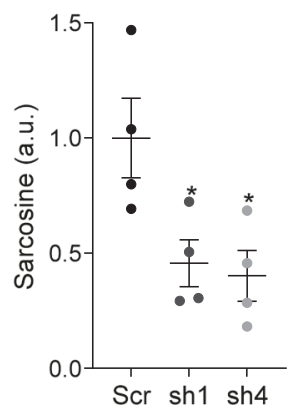

DU145 shGNMT

Fig. 4 Impact of genetic Gnmt modulation in prostate cancer pathogenesis. A-D, Effect of GNMT silencing on mRNA levels (A), cell number (B), foci formation (C), and sarcosine levels by LC/MS (D) in PC3 cells $(n=3)$. E-H, Effect of GNMT silencing on mRNA levels (E), cell number (F), foci formation $(\mathbf{G})$, and sarcosine levels by LC/MS $(\mathbf{H})$ in DU145 cells $(n=3-4)$. Data are represented as mean with SEM. scr: scramble short hairpin; sh1 and sh4: two different short-hairpins targeting GNMT; $p, p$ value; ${ }^{*} p<0.05 ;{ }^{* *} p<0.01 ;{ }^{* * *} p<0.001$. Statistic test: One Sample $(\mathbf{A}$, C, E, G) and or one-tailed paired (B, D, F, H) Student $T$-test was used for data analysis.

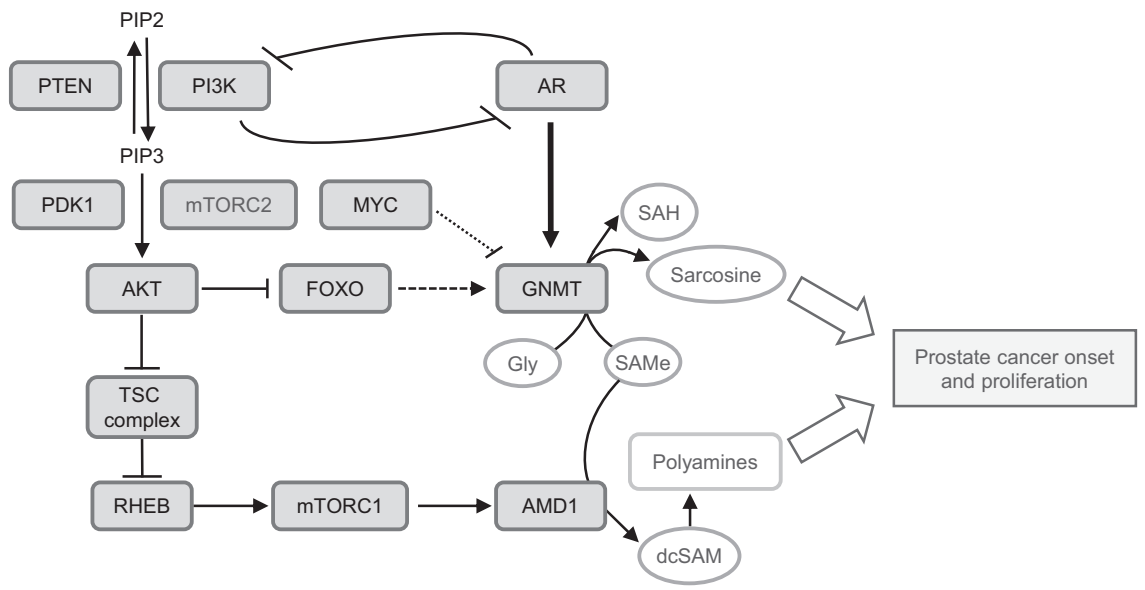

Fig. 5 Integrative view of GNMT regulation in prostate cancer. Schematic representation of Methionine cycle, Polyamine metabolism, and PI3K-mTOR axis interaction. PIP2 phosphatidylinositol 4,5-bisphosphate, PIP3 phosphatidylinositol 3,4,5-trisphosphate, PDK1 Phosphoinositide-dependent kinase 1, TSC tuberous sclerosis protein complex, RHEB Ras homolog enriched in brain, Gly Glycine.

iBET. On the other hand, we documented the negative regulation of GNMT mRNA levels by the PI3K pathway. PI3K activation, prominently due to alterations in PTEN, is a relevant oncogenic signal in cancer, and specifically in prostate tumors $[1,8,14]$ (Fig. 5). The fact that our mouse model exhibited an unexpected reduction in Gnmt expression prompted us to study the relationship between this event and the genetic nature of the tumors, driven by prostate-specific activation of the PI3K pathway. Our analysis in the mouse model, complemented with pharmacological analyses and associative studies in $\mathrm{PCa}$ specimens demonstrates that this metabolic enzyme is under negative regulation of the PI3K axis. We ruled out that this event would be mTORC1-dependent, in contrast to our observations with 
AMD1, another enzyme associated to SAMe metabolism [9]. Interestingly, PI3K and AKT inhibitors incremented GNMT expression, suggesting that the regulation stems from an AKT effector. There are two possible explanations for this observation. First, the PI3K and AR exhibit a mutually inhibitory cross talk $[47,48]$. It is therefore plausible that inhibition of PI3K-AKT would further enhance AR activity and promote GNMT expression, as reported for MYC [20]. However, the fact that we could replicate the increase in GNMT with PI3K inhibitors in AR-negative cells (PC3) suggests that another mechanism exists beyond the regulation by AR. Second, recent studies in different experimental systems suggest that FOXO (a transcription factor under negative regulation by AKT) regulates the expression of GNMT $[40,41]$. These studies are consistent with data generated in the context of ENCODE consortium, where FOXO1 is shown to bind to GNMT promoter region in HepG2 cells [49]. In this line, we confirmed the transcriptional control of GNMT by FOXO1 using shRNA against the transcription factor in prostate cancer cells. This mechanism is consistent with our results in vitro and in vivo. Altogether, our data reveal that GNMT expression is determined by the equilibrium between androgen and PI3K-dependent signals.

Our results provide further demonstration for the requirement of GNMT in the acquisition of cancerous features by prostate cells. To date, the association of GNMT to PCa biology is based on the use of cell lines and correlative human analyses [14, 15, 19]. We provide unprecedented evidence showing that expression of GNMT is required for the onset of invasive prostate carcinoma. Ablation of Gnmt in mice with conditional deletion of Pten in the prostate epithelium prevented the development of full-blown prostate carcinoma. Our results suggest that despite the PI3Kdependent downregulation of Gnmt in the murine prostate, basal expression of this gene is essential for PCa to emerge.

GNMT catalyzes the production of sarcosine and S-adenosylhomocysteine (SAH) from glycine and SAMe [12]. It has been proposed that sarcosine production accounts for the majority of pro-tumorigenic activities of GNMT [17]. In our study, sarcosine is the metabolite that best illustrates the changes in GNMT expression, which supports the notion that this metabolite could be responsible for its effects $[15,16,18]$. Sarcosine production is a suggested noninvasive PCa biomarker [15]. However, these results remain controversial, according to various follow-up studies in different $\mathrm{PCa}$ cohorts [50]. Moreover, many groups have attempted the development of methods to monitor sarcosine abundance [51-53], and combination of sarcosine with other metabolites has been tested in order to test its prognostic potential [54]. The opposed regulation of GNMT expression by AR vs. MYC and PI3K would suggest that the levels of sarcosine in PCa patients could be influenced by PTEN and MYC status. Therefore, it remains to be evaluated whether the potential of sarcosine as a PCa biomarker would benefit from PTEN and/or MYC status-based patient stratification.

PI3K activation elicits the concomitant repression of GNMT and activation of AMD1 [9]. This dual regulation suggests that the oncogenic signaling pathway could control the fate of SAMe by downregulating its demethylation in favor of its decarboxylation. Interestingly, GNMT perturbation alone does not affect polyamine biosynthesis based on our analysis of murine specimens and cell lines. These data is in line with the lack of oncogenic prostate phenotype observed in the mouse model upon Gnmt deletion alone, which would be expected if polyamine production would increase.

This study provides evidence of the requirement of GNMT for PCa pathogenesis, and reveals an unprecedented PI3K-dependent regulation that broadens our perspective around the prostateintrinsic signals that govern SAMe metabolism.

\section{REFERENCES}

1. Kypta $R$, Unda M, Carracedo A. Is the bench getting closer to the bedside in the war on cancer? A quick look at prostate cancer. Front Endocrinol. 2012;3:53.
2. Crawford ED, Schellhammer PF, McLeod DG, Moul JW, Higano CS, Shore N, et al. Androgen Receptor Targeted Treatments of Prostate Cancer: 35 Years of Progress with Antiandrogens. J Urol. 2018;200:956-66.

3. Grimm P, Billiet I, Bostwick D, Dicker AP, Frank S, Immerzeel J, et al. Comparative analysis of prostate-specific antigen free survival outcomes for patients with low, intermediate and high risk prostate cancer treatment by radical therapy. Results Prostate Cancer Results Study Group BJU Int. 2012;109(Suppl 1):22-29.

4. Abida W, Armenia J, Gopalan A, Brennan R, Walsh M, Barron D, et al. Prospective Genomic Profiling of Prostate Cancer Across Disease States Reveals Germline and Somatic Alterations That May Affect Clinical Decision Making. JCO Precis Oncol. 2017;2017:PO.17.00029.

5. Armenia J, Wankowicz SAM, Liu D, Gao J, Kundra R, Reznik E, et al. The long tail of oncogenic drivers in prostate cancer. Nat Genet. 2018;50:645-51.

6. Cancer Genome Atlas Research N. The Molecular Taxonomy of Primary Prostate Cancer. Cell. 2015;163:1011-25.

7. Chen Z, Trotman LC, Shaffer D, Lin HK, Dotan ZA, Niki M, et al. Crucial role of p53dependent cellular senescence in suppression of Pten-deficient tumorigenesis. Nature. 2005;436:725-30.

8. Nardella C, Carracedo A, Salmena L, Pandolfi PP. Faithfull modeling of PTEN loss driven diseases in the mouse. Curr Top Microbiol Immunol. 2011;347:135-68.

9. Zabala-Letona A, Arruabarrena-Aristorena A, Martin-Martin N, Fernandez-Ruiz S, Sutherland JD, Clasquin M, et al. mTORC1-dependent AMD1 regulation sustains polyamine metabolism in prostate cancer. Nature. 2017;547:109-13.

10. Song MS, Salmena L, Pandolfi PP. The functions and regulation of the PTEN tumour suppressor. Nat Rev Mol Cell Biol. 2012;13:283-96.

11. Arruabarrena-Aristorena A, Zabala-Letona A, Carracedo A. Oil for the cancer engine: The cross-talk between oncogenic signaling and polyamine metabolism. Sci Adv. 2018;4:eaar2606.

12. Lu SC, Mato JM. S-adenosylmethionine in liver health, injury, and cancer. Physiol Rev. 2012;92:1515-42.

13. Martinez-Chantar ML, Vazquez-Chantada M, Ariz U, Martinez N, Varela M, Luka Z, et al. Loss of the glycine $\mathrm{N}$-methyltransferase gene leads to steatosis and hepatocellular carcinoma in mice. Hepatology. 2008;47:1191-9.

14. Song YH, Shiota M, Kuroiwa K, Naito S, Oda Y. The important role of glycine $\mathrm{N}$-methyltransferase in the carcinogenesis and progression of prostate cancer. Mod Pathol. 2011;24:1272-80.

15. Sreekumar A, Poisson LM, Rajendiran TM, Khan AP, Cao Q, Yu J, et al. Metabolomic profiles delineate potential role for sarcosine in prostate cancer progression. Nature. 2009;457:910-4.

16. Heger Z, Merlos Rodrigo MA, Michalek P, Polanska H, Masarik M, Vit V, et al. Sarcosine Up-Regulates Expression of Genes Involved in Cell Cycle Progression of Metastatic Models of Prostate Cancer. PLoS One. 2016;11:e0165830.

17. Khan AP, Rajendiran TM, Ateeq B, Asangani IA, Athanikar JN, Yocum AK, et al. The role of sarcosine metabolism in prostate cancer progression. Neoplasia. 2013;15:491-501.

18. Strmiska V, Michalek P, Lackova Z, Guran R, Krizkova S, Vanickova L, et al. Sarcosine is a prostate epigenetic modifier that elicits aberrant methylation patterns through the SAMe-Dnmts axis. Mol Oncol. 2019;13:1002-17.

19. Huang YC, Lee CM, Chen M, Chung MY, Chang YH, Huang WJ, et al. Haplotypes, loss of heterozygosity, and expression levels of glycine $\mathrm{N}$-methyltransferase in prostate cancer. Clin Cancer Res. 2007;13:1412-20.

20. Barfeld SJ, Urbanucci A, Itkonen HM, Fazli L, Hicks JL, Thiede B, et al. c-Myc Antagonises the Transcriptional Activity of the Androgen Receptor in Prostate Cancer Affecting Key Gene Networks. EBioMedicine. 2017;18:83-93.

21. Lee $\mathrm{CM}$, Yen $\mathrm{CH}$, Tzeng TY, Huang $\mathrm{YZ}$, Chou $\mathrm{KH}$, Chang $\mathrm{TJ}$, et al. Androgen response element of the glycine $\mathrm{N}$-methyltransferase gene is located in the coding region of its first exon. Biosci Rep. 2013;33:e00070.

22. Ottaviani S, Brooke GN, O'Hanlon-Brown C, Waxman J, Ali S, Buluwela L. Characterisation of the androgen regulation of glycine $\mathrm{N}$-methyltransferase in prostate cancer cells. J Mol Endocrinol. 2013;51:301-12.

23. Chen M, Huang YL, Huang YC, Shui IM, Giovannucci E, Chen YC, et al. Genetic polymorphisms of the glycine $\mathrm{N}$-methyltransferase and prostate cancer risk in the health professionals follow-up study. PLoS One. 2014;9:e94683.

24. Ugalde-Olano A, Egia A, Fernandez-Ruiz S, Loizaga-Iriarte A, Zuniga-Garcia $P$, Garcia S, et al. Methodological aspects of the molecular and histological study of prostate cancer: focus on PTEN. Methods. 2015;77-78:25-30.

25. Torrano V, Valcarcel-Jimenez L, Cortazar AR, Liu X, Urosevic J, Castillo-Martin M, et al. The metabolic co-regulator PGC1alpha suppresses prostate cancer metastasis. Nat Cell Biol. 2016;18:645-56.

26. Camacho L, Zabala-Letona A, Cortazar AR, Astobiza I, Dominguez-Herrera A, Ercilla A, et al. Identification of Androgen Receptor Metabolic Correlome Reveals the Repression of Ceramide Kinase by Androgens. Cancers.2021;13:4307.

27. Serra H, Chivite I, Angulo-Urarte A, Soler A, Sutherland JD, ArruabarrenaAristorena $A$, et al. PTEN mediates Notch-dependent stalk cell arrest in angiogenesis. Nat Commun. 2015;6:7935. 
28. Carracedo A, Ma L, Teruya-Feldstein J, Rojo F, Salmena L, Alimonti A, et al. Inhibition of mTORC1 leads to MAPK pathway activation through a PI3K-dependent feedback loop in human cancer. J Clin Invest. 2008;118:3065-74.

29. Carracedo A, Weiss D, Leliaert AK, Bhasin M, de Boer VC, Laurent G, et al. A metabolic prosurvival role for PML in breast cancer. J Clin Investig. 2012;122:3088-3100.

30. Jha AK, Huang SC, Sergushichev A, Lampropoulou V, Ivanova Y, Loginicheva E, et al. Network integration of parallel metabolic and transcriptional data reveals metabolic modules that regulate macrophage polarization. Immunity. 2015;42:419-30.

31. Melamud E, Vastag L, Rabinowitz JD. Metabolomic analysis and visualization engine for LC-MS data. Anal Chem. 2010;82:9818-26.

32. Hahne F, Ivanek R. Visualizing Genomic Data Using Gviz and Bioconductor. Methods Mol Biol. 2016;1418:335-51.

33. Edgar R, Domrachev M, Lash AE. Gene Expression Omnibus: NCBI gene expres sion and hybridization array data repository. Nucleic Acids Res. 2002;30:207-10.

34. Cerami E, Gao J, Dogrusoz U, Gross BE, Sumer SO, Aksoy BA, et al. The cBio cancer genomics portal: an open platform for exploring multidimensional cancer genomics data. Cancer Disco. 2012;2:401-4.

35. Gao J, Aksoy BA, Dogrusoz U, Dresdner G, Gross B, Sumer SO, et al. Integrative analysis of complex cancer genomics and clinical profiles using the cBioPortal. Sci Signal. 2013;6:pl1.

36. Varambally S, Yu J, Laxman B, Rhodes DR, Mehra R, Tomlins SA, et al. Integrative genomic and proteomic analysis of prostate cancer reveals signatures of metastatic progression. Cancer Cell. 2005;8:393-406.

37. Barrett $T$, Wilhite SE, Ledoux $P$, Evangelista C, Kim IF, Tomashevsky M, et al. NCBI GEO: archive for functional genomics data sets-update. Nucleic Acids Res. 2012;41:D991-D995.

38. Taylor BS, Schultz N, Hieronymus H, Gopalan A, Xiao Y, Carver BS, et al. Integrative genomic profiling of human prostate cancer. Cancer Cell. 2010;18:11-22.

39. Hay N. Interplay between FOXO, TOR, and Akt. Biochim Biophys Acta. 2011;1813:1965-70.

40. Hwang I, Uchida H, Dai Z, Li F, Sanchez T, Locasale JW, et al. Cellular stress signaling activates type-I IFN response through FOXO3-regulated lamin posttranslational modification. Nat Commun. 2021;12:640.

41. Obata F, Kuranaga $E$, Tomioka $\mathrm{K}$, Ming $\mathrm{M}$, Takeishi $\mathrm{A}$, Chen $\mathrm{CH}$, et al. Necrosisdriven systemic immune response alters SAM metabolism through the FOXOGNMT axis. Cell Rep. 2014;7:821-33.

42. Dunham I, Kundaje A, Aldred SF, Collins PJ, Davis CA, Doyle F, et al. An integrated encyclopedia of DNA elements in the human genome. Nature. 2012;489:57-74.

43. Cortazar AR, Torrano V, Martin-Martin N, Caro-Maldonado A, Camacho L, Hermanova I, et al. CANCERTOOL: A Visualization and Representation Interface to Exploit Cancer Datasets. Cancer Res. 2018;78:6320-8.

44. Tyekucheva S, Bowden M, Bango C, Giunchi F, Huang Y, Zhou C, et al. Stromal and epithelial transcriptional map of initiation progression and metastatic potential of human prostate cancer. Nat Commun. 2017;8:420.

45. Delmore JE, Issa GC, Lemieux ME, Rahl PB, Shi J, Jacobs HM, et al. BET bromodomain inhibition as a therapeutic strategy to target c-Myc. Cell. 2011;146:904-17.

46. DebRoy S, Kramarenko II, Ghose S, Oleinik NV, Krupenko SA, Krupenko NI. A novel tumor suppressor function of glycine $\mathrm{N}$-methyltransferase is independent of its catalytic activity but requires nuclear localization. PLoS One. 2013;8:e70062.

47. Carver BS, Chapinski C, Wongvipat J, Hieronymus H, Chen Y, Chandarlapaty S, et al. Reciprocal feedback regulation of $\mathrm{PI} 3 \mathrm{~K}$ and androgen receptor signaling in PTEN-deficient prostate cancer. Cancer Cell. 2011;19:575-86.

48. Mulholland DJ, Tran LM, Li Y, Cai H, Morim A, Wang S, et al. Cell autonomous role of PTEN in regulating castration-resistant prostate cancer growth. Cancer Cell. 2011;19:792-804

49. Consortium EP. The ENCODE (ENCyclopedia Of DNA Elements) Project. Science 2004;306:636-40.

50. Lima AR, Bastos Mde L, Carvalho M, Guedes de Pinho P. Biomarker Discovery in Human Prostate Cancer: an Update in Metabolomics Studies. Transl Oncol. 2016;9:357-70.

51. Pacik D, Plevova M, Urbanova L, Lackova Z, Strmiska V, Necas A, et al. Identification of Sarcosine as a Target Molecule for the Canine Olfactory Detection of Prostate Carcinoma. Sci Rep. 2018;8:4958.

52. Pundir CS, Deswal R, Kumar P. Quantitative analysis of sarcosine with special emphasis on biosensors: a review. Biomarkers. 2019:24:415-22.

53. Yamkamon V, Yee PP, Yainoi S, Eiamphungporn W, Suksrichavalit T. Simultaneous determination of sarcosine and its related metabolites by gas chromatographytandem mass spectrometry for prostate cancer diagnosis. EXCLI J. 2018;17:965-79.

54. Wang M, Zou L, Liang J, Wang X, Zhang D, Fang Y, et al. The Urinary Sarcosine/ Creatinine Ratio is a Potential Diagnostic and Prognostic Marker in Prostate Cancer, Med Sci Monit. 2018;24:3034-41.

\section{ACKNOWLEDGEMENTS}

We are grateful to the Carracedo lab for valuable input, to Drs. Ana M. Aransay, James D. Sutherland and F. Elortza for technical advice, and Drs. Michelle Clasquin, Katie Sellers and Katya Marjon at Agios Pharmaceuticals for performing, processing and analyzing the metabolomics experiments. We thank the Basque Biobank for Research (BIOEF) for the support with prostate specimen acquisition and management. A.A-A. was funded by the Basque Government (predoctoral fellowship). V.T. is funded by Fundación Vasca de Innovación e Investigación Sanitarias, BIOEF (BIO15/CA/052), the AECC J.P. Bizkaia, the Basque Department of Health (2016111109) and the MICINN RTI2018-097267-B-I00. I.M. is supported by Fundación Cris Contra el Cáncer (PR_TPD_2020-19). The work of A. Carracedo is supported by the Basque Department of Industry, Tourism and Trade (Elkartek), the department of education (IKERTALDE IT1106-16) and health (RIS3), the BBVA foundation, the MICINN (SAF2016-79381-R PID2019-108787RB-I00 (FEDER/EU); Severo Ochoa Excellence Accreditation SEV-20160644; Excellence Networks RED2018-102769-T), the AECC (GCTRA18006CARR), Vencer el Cáncer Foundation, La Caixa Foundation (ID 100010434), under the agreement LCF/PR/HR17/ and the European Research Council (Starting Grant 336343, PoC 754627, Consolidator Grant 819242). CIBERONC was co-funded with FEDER funds and funded by ISCIII. We are grateful for the support of Mondravember and Movembergara. A.E. was supported by MCIN/AEI/10.13039/501100011033 and the EU programme NextGenerationEU/PRTR (IJC2020-043583-I). The work of JM Mato was supported by NIH grant R01CA172086 and SAF2017-88041-R. EB is funded by the MICINN (BFU2016-76872-R (FEDER/EU), PID2019-108112RB-I00, and Excellence Networks SAF2017-90794-REDT).

\section{AUTHOR CONTRIBUTIONS}

$A Z$ and $A A-A$ performed the majority of in vitro and in vivo assays and performed the data analysis. SF-R, OC, AE, AM, TM, LC, MP-V, PS-M, VT, NM-M, CV, and PZ-G provided technical support with in vitro experiments. IM provided bioinformatics support. MC$\mathrm{M}$ performed the pathological analysis of mouse prostates. AU-O performed the pathological analysis of human prostate specimens. AL-I and MU recruited the prostate cancer and hyperplasia patients for the analysis. JMM and MLM-C provided support with the Gnmt deficient mouse model. EB supervised the work of TM and provided support in the revision and discussion of the manuscript. AC conceived the study, supervised the execution of the project and wrote the manuscript.

\section{COMPETING INTERESTS}

The authors declare no competing interests.

\section{ADDITIONAL INFORMATION}

Supplementary information The online version contains supplementary material available at https://doi.org/10.1038/s41389-022-00382-x.

Correspondence and requests for materials should be addressed to Arkaitz Carracedo.

Reprints and permission information is available at http://www.nature.com/ reprints

Publisher's note Springer Nature remains neutral with regard to jurisdictional claims in published maps and institutional affiliations.

\footnotetext{
Open Access This article is licensed under a Creative Commons Attribution 4.0 International License, which permits use, sharing, adaptation, distribution and reproduction in any medium or format, as long as you give appropriate credit to the original author(s) and the source, provide a link to the Creative Commons license, and indicate if changes were made. The images or other third party material in this article are included in the article's Creative Commons license, unless indicated otherwise in a credit line to the material. If material is not included in the article's Creative Commons license and your intended use is not permitted by statutory regulation or exceeds the permitted use, you will need to obtain permission directly from the copyright holder. To view a copy of this license, visit http://creativecommons. org/licenses/by/4.0/.
}

(c) The Author(s) 2022 